Fabrici, Igor; Jendrol', Stanislav:

An inequality concerning edges of minor weight in convex 3-polytopes

URN: $\quad$ urn:nbn:de:gbv:ilm1-2020200023

\begin{tabular}{ll}
\hline Original published in: & $\begin{array}{l}\text { Discussiones mathematicae. Graph theory / Uniwersytet Zielonogórski, } \\
\text { Wydział Matematyki, Informatyki i Ekonometrii. - Warsaw : De Gruyter } \\
\end{array}$ \\
& Open. - 16 (1996), 1, p. 81-87. \\
& 1996 \\
Original published: & $2083-5892$ \\
ISSN: & $\begin{array}{l}10.7151 / \text { dmgt.1024 } \\
\text { DOI: }\end{array}$ \\
[Visited: & This work is licensed under a Creative Commons Attribution- \\
& $\begin{array}{l}\text { NonCommercial-NoDerivatives 3.0 Unported license. } \\
\text { To view a copy of this license, visit } \\
\text { http://creativecommons.org/licenses/BY-NC-ND/3.0/ }\end{array}$ \\
\hline
\end{tabular}


Discussiones Mathematicae

Graph Theory 16(1996) 81-87

\title{
AN INEQUALITY CONCERNING EDGES OF MINOR WEIGHT IN CONVEX 3-POLYTOPES
}

\author{
IGOR FABRICI \\ Institute of Mathematics, Technical University Ilmenau \\ PF 327, D-98684 Ilmenau, Germany \\ e-mail: fabrici@mathematik.tu-ilmenau.de \\ and \\ StAnislaV JendRol' \\ Department of Geometry and Algebra, P.J. Šafárik University \\ Jesenná 5, 04154 Košice, Slovak Republic \\ e-mail: jendrol@kosice.upjs.sk
}

Dedicated to Professor E. Jucovič on the occasion of his 70th birthday.

\begin{abstract}
Let $e_{i j}$ be the number of edges in a convex 3-polytope joining the vertices of degree $i$ with the vertices of degree $j$. We prove that for every convex 3 -polytope there is $20 e_{3,3}+25 e_{3,4}+16 e_{3,5}+10 e_{3,6}+$ $6 \frac{2}{3} e_{3,7}+5 e_{3,8}+2 \frac{1}{2} e_{3,9}+2 e_{3,10}+16 \frac{2}{3} e_{4,4}+11 e_{4,5}+5 e_{4,6}+1 \frac{2}{3} e_{4,7}+5 \frac{1}{3} e_{5,5}+$ $2 e_{5,6} \geq 120$; moreover, each coefficient is the best possible. This result brings a final answer to the conjecture raised by B. Grünbaum in 1973.
\end{abstract}

Keywords: planar graph, convex 3-polytope, normal map.

1991 Mathematics Subject Classification: 52B10, 52B05, 05C10.

\section{Introduction and Statement of Results}

This note deals with connected planar maps. We use standard terminology and notation of graph theory, see e.g. Ore [16]. We recall, however, more specialized notions. A plane map is called normal if it contains neither vertices nor faces incident with less than 3 edges. Notice, however, that both loops and multiple edges can appear in a normal plane map. By the 
Steinitz's theorem (see e.g. Grünbaum [11], Jucovič [13]) convex 3-polytopes are distinguished among all planar maps by the property that their graphs are 3-connected. The degree of a face $\omega$ is the number of edges incident to $\omega$ where each cut-edge is counted twice. Similarly, each loop contributes 2 to the degree of the incident vertex. Vertices and faces of degree $i$ are called $i$-vertices and $i$-faces, respectively. Let $e_{i, j}(M)=e_{i, j}$ be the number of edges in a planar map $M$ which join $i$-vertices and $j$-vertices. Recall that a convex 3-polytope is called simplicial if all its faces are 3 -gons.

An excellent theorem of Kotzig [14] (see also [1,3,6,7,8,13,15,]) states that every convex 3 -polytope contains an edge of the weight (i.e., the sum of degrees of its endvertices) at most 13 ; in other words $\sum_{i+j \leq 13} e_{i, j}>0$. This Kotzig's result was further developed in various directions, see e.g. Borodin [1,2,3], Grünbaum [6,7,8], Grünbaum and Shephard [9], Ivančo [10], Ivančo and Jendrol' [11], Jucovič [12,13], Zaks [17].

Grünbaum [8] has brought an idea that a relation of the type $\sum_{i+j \leq 13} \alpha_{i, j} e_{i, j} \geq 1$ should hold for each convex 3 -polytope $\left(\alpha_{i, j}\right.$ denotes the coeficient at $e_{i j}$ ) and has conjectured that the following holds for every simplicial convex 3-polytope

$$
\begin{aligned}
20 e_{3,3} & +15 e_{3,4}+12 e_{3,5}+10 e_{3,6}+6 \frac{2}{3} e_{3,7}+5 e_{3,8}+3 \frac{1}{3} e_{3,9}+2 e_{3,10} \\
& +12 e_{4,4}+7 e_{4,5}+5 e_{4,6}+4 e_{4,7}+2 \frac{2}{3} e_{4,8}+\frac{2}{3} e_{4,9} \\
& +4 e_{5,5}+2 e_{5,6}+\frac{1}{3} e_{5,7} \\
& +12 e_{6,6} \geq 120 .
\end{aligned}
$$

Jucovič [12] proved that for each simplicial convex 3-polytope there is

$$
\begin{aligned}
20 e_{3,3} & +25 e_{3,4}+16 e_{3,5}+10 e_{3,6}+6 \frac{2}{3} e_{3,7}+5 e_{3,8}+2 \frac{1}{2} e_{3,9}+2 e_{3,10} \\
& +20 e_{4,4}+11 e_{4,5}+5 e_{4,6}+6 e_{4,7}+5 e_{4,8}+3 e_{4,9} \\
& +8 e_{5,5}+2 e_{5,6}+2 e_{5,7}+2 e_{5,8} \geq 120 .
\end{aligned}
$$

Later on Jucovič, in [13], proved that this inequality holds for all convex 3 -polytopes.

For a wider class of planar maps which also includes convex 3-polytopes Borodin [3] has obtained.

Theorem 1. For each normal planar map there holds

$$
\begin{aligned}
40 e_{3,3} & +25 e_{3,4}+16 e_{3,5}+10 e_{3,6}+6 \frac{2}{3} e_{3,7}+5 e_{3,8}+2 \frac{1}{2} e_{3,9}+2 e_{3,10} \\
& +16 \frac{2}{3} e_{4,4}+11 e_{4,5}+5 e_{4,6}+1 \frac{2}{3} e_{4,7} \\
& +5 \frac{1}{3} e_{5,5}+2 e_{5,6} \geq 120
\end{aligned}
$$

moreover, each coefficient of this inequality is the best possible. 
In the same paper Borodin [3] proves that for simplicial convex 3-polytopes (1) is the best possible if we put $\alpha_{3,3}=20$ instead of $\alpha_{3,3}=40$. For other results of this type see Borodin [1,2,3], Borodin and Sanders [5], Jucovič [13].

The main purpose of the present note is to give a final answer to the above mentioned conjecture by Grünbaum [8]. We prove the following

Theorem 2. For each convex 3-polytopes there holds

$$
\begin{aligned}
20 e_{3,3} & +25 e_{3,4}+16 e_{3,5}+10 e_{3,6}+6 \frac{2}{3} e_{3,7}+5 e_{3,8}+2 \frac{1}{2} e_{3,9}+2 e_{3,10} \\
& +16 \frac{2}{3} e_{4,4}+11 e_{4,5}+5 e_{4,6}+1 \frac{2}{3} e_{4,7} \\
& +5 \frac{1}{3} e_{5,5}+2 e_{5,6} \geq 120
\end{aligned}
$$

moreover, each coefficient of this inequality is the best possible.

\section{Proof of Theorem 2}

We prove our Theorem 2 in a dual form. It is well known that the dual of a 3-connected planar map is also 3-connected, see e.g. Ore [16, Chapter 3] and, due to Steinitz's theorem, it is also true for convex 3-polytopes. It is easy to check that the dual of a normal map is again normal.

For the purposes of this proof an edge $h$ is called an $(i, j)$-edge when it is incident with an $i$-gon and a $j$-gon. Let $g_{i, j}(M)=g_{i j}$ denote the number of $(i, j)$-edges in a map $M$. If $M^{d}$ is the dual to a normal map $M$, then clearly $e_{i j}\left(M^{d}\right)=g_{i j}(M)$. Let $V(M), E(M)$ and $F(M)$ denote the set of vertices, edges and faces of the map $M$, respectively.

The proof is by contradiction. Replace $e_{i j}$ with $g_{i j}$ in the left part of (2) and denote it by $\sum$. We want to prove that for every 3 -connected planar $M$ there is $\sum(M) \geq 120$. Suppose $M$ be a counterexample having a minimum number of faces.

To obtain a contradiction we are going to look for a suitable configuration in $M$ which will be changed locally to obtain a new 3-connected plane map $M^{*}$ with $\sum\left(M^{*}\right) \leq \sum(M)<120$ and with a fewer number of faces than in $M$. During this transformation of the map $M$ into $M^{*}$ some edges and vertices of $M$ are deleted, some edges change their types (an edge is of the type $(i, j)$ if it is an $(i, j)$-edge) and some new edges and vertices can appear in $M^{*}$.

Asociate with an $(i, j)$-edge $h$ of the map $M$ the charge $\alpha(h, M)=\alpha_{i j}$, where $\alpha_{i j}$ is as in (2) or $\alpha_{i, j}=0$ for $i=3, j \geq 11$ or $i=4, j \geq 8$ or 
$i=5, j \geq 7$ or $i \geq 6, j \geq 6$. Hence $\sum(M)=\sum_{h \in E(M)} \alpha(h, M)$. Let $\Delta(h)=\alpha(h, M)-\alpha\left(h, M^{*}\right)$.

Since every 3-connected plane map is also normal Theorem 1 yields $g_{3,3}(M) \neq 0$, i.e., $M$ contains a $(3,3)$-edge $h_{0}=u v$. Denote by $s$ and $t$ the vertices incident to triangles incident with $h_{0}$ and different from $u$ and $v$, see Figure 1. Let $h_{1}=u s, h_{2}=s v, h_{3}=v t$ and $h_{4}=u t$ be edges of $M$.

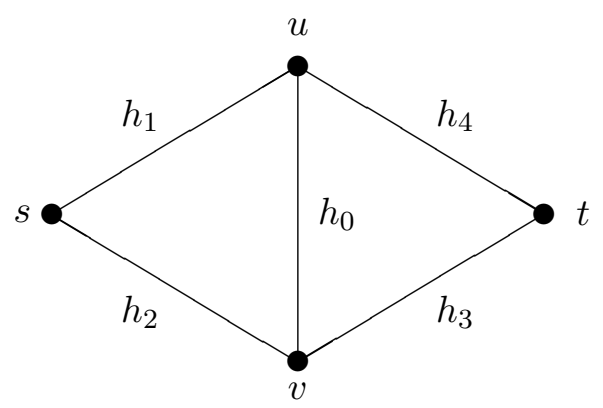

Figure 1

To finish our proof several cases have to be considered

Case 1. $\operatorname{deg} u \geq 4$ and $\operatorname{deg} v \geq 4$.

1.1. Let $\operatorname{deg} s=3$ or $\operatorname{deg} t=3$. The required map $M^{*}$ is obtained by deleting the edge $h_{0}$ from $M$, i.e., $M^{*}=M-h_{0}$. Because $M$ is 3 -connected and at least one of the vertices $s$ and $t$ is a 3 -vertex also $M^{*}$ is 3 -connected. We can easily see that $\left|F\left(M^{*}\right)\right|=|F(M)|-1$ and $\Delta\left(\sum\right)=\sum(M)-\sum\left(M^{*}\right)=$ $\alpha\left(h_{0}, M\right)+\sum_{i=1}^{4}\left(\alpha\left(h_{i}, M\right)-\alpha\left(h_{i}, M^{*}\right)\right)=\alpha_{3,3}+\sum_{i=1}^{4} \Delta\left(h_{i}\right) \geq 20+$ $4 \cdot(-5)=0$. The last inequality is due to the fact that if a $(3, k)$-edge $h$ is transformed into a $(4, k)$-edge, its charge always decreases or is the same except of the case $k=3$. We also refer to the fact that $\Delta\left(h_{i}\right) \geq-5$ for any edge $h_{i} \in E(M)$.

1.2. $\operatorname{deg} s \geq 4$ and $\operatorname{deg} t \geq 4$. In this case we transform $M$ into $M^{*}$ as shown in Figure 2. We delete the edge $h_{0}$ from $M$ and split the vertex $t$ of $M$ into two new vertices $t_{1}$ and $t_{2}$ such that we obtain, in $M^{*}, \operatorname{deg} t_{1}=3$ and $\operatorname{deg} t_{2}=\operatorname{deg} t-1$. (The reason for this transformation of $M$ into $M^{*}$ is to preserve 3 -connectivity also in $M^{*}$.) Let $h^{\prime}, h_{1}, h_{2}, h_{3}$ and $h_{4}$ be edges and $\omega_{1}$ and $\omega_{2}$ be faces of $M^{*}$ as depicted in Figure 2. Without loss of generality we can assume that $4 \leq \operatorname{deg} \omega_{1} \leq \operatorname{deg} \omega_{2}$. Put $\Delta^{*}=\sum_{x \in E(M)-\left\{h_{0}, h_{3}, h_{4}\right\}} \Delta(x)$. Then we have $\left|F\left(M^{*}\right)\right|=|F(M)|-1$ and $\Delta\left(\sum\right)=\alpha\left(h_{0}, M\right)+\Delta\left(h_{3}\right)+$ 
$\Delta\left(h_{4}\right)+\Delta^{*}-\alpha\left(h^{\prime}, M^{*}\right) \geq 0$. To check it use $\alpha\left(h_{0}, M\right)=\alpha_{3,3}=20$ and for the values $\Delta\left(h_{3}\right), \Delta\left(h_{4}\right), \alpha\left(h^{\prime}, M^{*}\right)$ and $\Delta^{*}$ see Table 1 below. To count $\Delta^{*}$ we also refer to the fact that $g_{3,3}(M) \leq 5$ (because $M$ is a counterexample) and consider the "worst" case.

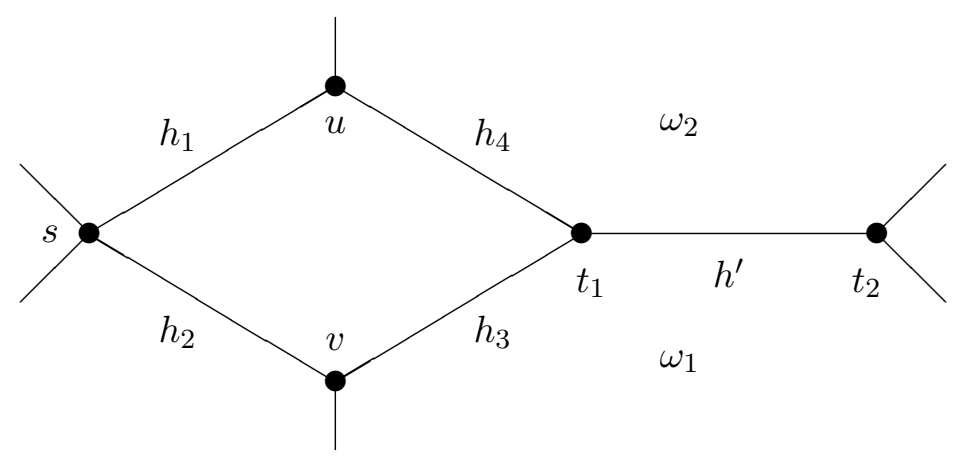

Figure 2

Case 2. $\operatorname{deg} u=3$ and $\operatorname{deg} v \geq 4$.

Let $w$ be a face incident to the edges $h_{1}$ and $h_{4}$, see Figure 1 .

2.1. If $\operatorname{deg} w=3$ then $M^{*}$ is obtained by removing the vertex $u$ from $M$, i.e. $M^{*}=M-\{u\}$. We have $\Delta\left(\sum\right)=\alpha\left(h_{0}, M\right)+\alpha\left(h_{1}, M\right)+\alpha\left(h_{4}, M\right)=60>0$ and $\left|F\left(M^{*}\right)\right|=|F(M)|-2$.

2.2. Let $\operatorname{deg} w=k \geq 4$. If we delete the vertex $u$ from $M$ and then insert a new edge $h^{*}=s t$ we obtain a required map $M^{*}, M^{*}=M-\{u\}+\left\{h^{*}\right\}$. In this case $\left|F\left(M^{*}\right)\right|=|F(M)|-1$ and we can check that $\Delta\left(\sum\right)=\alpha\left(h_{0}, M\right)+$ $\alpha\left(h_{1}, M\right)+\alpha\left(h_{4}, M\right)-\alpha\left(h^{*}, M^{*}\right)+\tilde{\Delta} \geq 0$. To see it, take $\alpha\left(h_{0}, M\right)=$ $\alpha_{3,3}=20$ and the values $\alpha\left(h_{1}, M\right), \alpha\left(h_{4}, M\right), \alpha\left(h^{*}, M^{*}\right)$ and $\tilde{\Delta}$ from the Table 2 below; here $\tilde{\Delta}=\sum \Delta(g)$, where the sum is taken over all edges $g$ incident to the face $\omega, g \neq h_{1}, h_{4}$. Note that during this transformation the edge $g$ changes its type $(n, k)$ into the type $(n, k-1)$ and in the counting we consider the worst case, that is $\tilde{\Delta} \geq(k-2)\left(\alpha_{3, k}-\alpha_{3, k-1}\right)$.

Case 3. $\operatorname{deg} u=\operatorname{deg} v=3$.

This assumption leads immediately to the graph of the tetrahedron or to a 2-connected planar map. In both cases we get a contradiction.

The proof that a 3 -connectivity of $M$ implies a 3-connectivity of $M^{*}$ is easy and is left to the reader. 
The coefficient $\alpha_{3,3}=20$ cannot be improved as we can see from the tetrahedron. The above mentioned examples by Borodin [3] also show the impossibility to improve the other coefficient $\alpha_{i, j}$ in Theorem 2 .

Table 1

\begin{tabular}{|c|c|c|c|c|c|}
\hline $\operatorname{deg} \omega_{1}$ & $\operatorname{deg} \omega_{2}$ & $\Delta\left(h_{3}\right)$ & $\Delta\left(h_{4}\right)$ & $\alpha\left(h^{\prime}, M^{*}\right)$ & $\Delta^{*} \geq$ \\
\hline 4 & 4 & $3 \frac{1}{3}$ & $3 \frac{1}{3}$ & $16 \frac{2}{3}$ & -10 \\
4 & 5 & $3 \frac{1}{3}$ & 14 & 11 & -20 \\
4 & 6 & $3 \frac{1}{3}$ & 11 & 5 & -20 \\
4 & 7 & $3 \frac{1}{3}$ & $8 \frac{1}{3}$ & $1 \frac{2}{3}$ & -20 \\
4 & $\geq 8$ & $3 \frac{1}{3}$ & $\geq 0$ & 0 & -20 \\
5 & 5 & 14 & 14 & $5 \frac{1}{3}$ & -10 \\
5 & 6 & 14 & 11 & 2 & -10 \\
5 & $\geq 7$ & 14 & $\geq 0$ & 0 & -10 \\
$\geq 6$ & $\geq 6$ & $\geq 0$ & $\geq 0$ & 0 & -10 \\
\hline
\end{tabular}

Table 2

\begin{tabular}{|c|c|c|c|c|}
\hline $\operatorname{deg} \omega$ & $d\left(h_{1}, M\right)$ & $d\left(h_{4}, M\right)$ & $d\left(h^{*}, M^{*}\right)$ & $\tilde{\Delta} \geq$ \\
\hline 4 & 25 & 25 & 20 & $2 \cdot\left(-8 \frac{1}{3}\right)$ \\
5 & 16 & 16 & 25 & $3 \cdot(-9)$ \\
6 & 10 & 10 & 16 & $4 \cdot(-6)$ \\
7 & $6 \frac{2}{3}$ & $6 \frac{2}{3}$ & 10 & $5 \cdot\left(-3 \frac{1}{3}\right)$ \\
8 & 5 & 5 & $6 \frac{2}{3}$ & $6 \cdot\left(-1 \frac{2}{3}\right)$ \\
9 & $2 \frac{1}{2}$ & $2 \frac{1}{2}$ & 5 & $7 \cdot\left(-2 \frac{1}{2}\right)$ \\
10 & 2 & 2 & $2 \frac{1}{2}$ & $8 \cdot\left(-\frac{1}{2}\right)$ \\
11 & 0 & 0 & 2 & $9 \cdot(-2)$ \\
$\geq 12$ & 0 & 0 & 0 & 0 \\
\hline
\end{tabular}




\section{REFERENCES}

[1] O. V. Borodin, Computing light edges in planar graphs, in: R. Bodendiek, R. Henn, eds., Topics in Combinatorics and Graph Theory (Physica-Verlag, Heidelberg, 1990) 137-144.

[2] O. V. Borodin, Structural properties and colorings of plane graphs, Ann. Discrete Math. 51 (1992) 31-37.

[3] O. V. Borodin, Precise lower bound for the number of edges of minor weight in planar maps, Math. Slovaca 42 (1992) 129-142.

[4] O. V. Borodin, Structural properties of planar maps with the minimal degree 5, Math. Nachr. 158 (1992) 109-117.

[5] O. V. Borodin and D. P. Sanders, On light edges and triangles in planar graph of minimum degree five, Math. Nachr. 170 (1994) 19-24.

[6] B. Grünbaum, Acyclic colorings of planar graphs, Israel J. Math. 14 (1973) 390-408.

[7] B. Grünbaum, Polytopal graphs, in: D. R. Fulkerson, ed., Studies in Graph Theory, MAA Studies in Mathematics 12 (1975) 201-224.

[8] B. Grünbaum, New views on some old questions of combinatorial geometry, Int. Teorie Combinatorie, Rome, 1973, 1 (1976) 451-468.

[9] B. Grünbaum and G. C. Shephard, Analogues for tiling of Kotzig's theorem on minimal weights of edges, Ann. Discrete Math. 12 (1982) 129-140.

[10] J. Ivančo, The weight of a graph, Ann. Discrete Math. 51 (1992) 113-116.

[11] J. Ivančo and S. Jendrol', On extremal problems concerning weights of edges of graphs, in: Coll. Math. Soc. J. Bolyai, 60. Sets, Graphs and Numbers, Budapest (Hungary) 1991 (North Holland, 1993) 399-410.

[12] E. Jucovič, Strengthening of a theorem about 3-polytopes, Geom. Dedicata 3 (1974) 233-237.

[13] E. Jucovič, Convex 3-polytopes (Veda, Bratislava, 1981, Slovak).

[14] A. Kotzig, Contribution to the theory of Eulerian polyhedra, Mat.-Fyz. Cas. SAV (Math. Slovaca) 5 (1955) 101-103 (Slovak; Russian summary).

[15] A. Kotzig, From the theory of Euler's polyhedra, Mat. Cas. (Math. Slovaca) 13 (1963) 20-34 (Russian).

[16] O. Ore, The four-color problem (Academic Press, New York, 1967).

[17] J. Zaks, Extending Kotzig's theorem, Israel J. Math. 45 (1983) 281-296.

Received 24 January 1996 Revised 15 April 1996 